Pacific Journal of Mathematic 


\section{ON THE NÖRLUND SUMMABILITY OF FOURIER SERIES}

\section{B. N. SAHNeY}

1. Let $f(x)$ be a function integrable $-L$ over the interval $(-\pi, \pi)$ and periodic with period $2 \pi$, outside this interval.

Let

$$
\phi(t)=\frac{1}{2}\{f(x+t)+f(x-t)-2 s(x)\},
$$

and

$$
\frac{1}{2} a_{0}+\sum_{n=1}^{\infty}\left(a_{n} \cos n x+b_{n} \operatorname{Sin} n x\right)
$$

be the Fourier Series of the function $\phi(t)$.

Nörlund Summability of Fourier Series (1.2) has been considered by Woronoi [6] and later on by Nörlund [4]. These results have been extended by Hille and Tamarkin [2], [3], and later on by Astrachan [1]. Recently, extending a result due to Hille and Tamarkin [3], Varshney [5] has proved the proved the following:

THEOREM. V. If the sequence $\left\{p_{n}\right\}$ satisfies the following conditions:

$$
\begin{gathered}
\frac{n|p n|}{\log n}<c\left|P_{n}\right|, \\
\sum_{k=0}^{n} \frac{k\left|p_{k}-p_{k-1}\right|}{\log (k+1)}<c\left|P_{n}\right|
\end{gathered}
$$

and

$$
\sum_{k=0}^{n} \frac{P_{k}}{k \log (k+1)}<c\left|P_{n}\right|
$$

and also if

$$
\bar{\Phi}_{1}(t)=\int_{0}^{t}|\phi(u)| d u=0\left(t / \log \frac{1}{t}\right)
$$

then the Fourier Series (1.2) associated with the function $\phi(t)$ is summable by Nörlund means i.e. summable $\left(N, p_{n}\right)$ to the sum zero at the point $t=x$.

The object here is to prove the following:

THEOREM. If the sequence $\left\{p_{n}\right\}$ satisfies the following conditions 


$$
\frac{n\left|p_{n}\right|}{(\log n)^{r}}<c\left|P_{n}\right|
$$

and

$$
\sum_{k=0}^{n} \frac{k\left|p_{k}-p_{k-1}\right|}{\{\log (k+1)\}^{r}}<c\left|P_{n}\right|
$$

and also if

$$
\bar{\Phi}_{1}(t)=\int_{0}^{t}|\phi(u)| d u=0\left\{t /\left(\log \frac{1}{t}\right)^{r}\right\}
$$

and

$$
\frac{1}{P(n)} \int_{\pi / n}^{\delta} \frac{|\phi(t+\pi / n)-\phi(t)|}{t} P\left(\frac{1}{t}\right) d t=0(1)
$$

then the Fourier Series (1.2) associated with the function $\phi(t)$ is summable by Nörlund means i.e. summable $\left(N, p_{n}\right)$ to the sum zero at the point $t=x$ for all $0 \leqq r \leqq 1$.

2. The following notations will be used in the sequel.

We write $S_{n}(x)$ as the $n$th partial sum of the series (1.2) and the Nörlund transform of the partial sum of the series (1.2) we denote by $\sigma_{n}(x)$.

Also we write; where $P_{n} \equiv P(n)$,

$$
N_{n}(t)=\frac{1}{\pi P_{n}} \sum_{k=0}^{n} p_{n-k} \frac{\operatorname{Sin}(k+1 / 2) t}{t} .
$$

We recall that the conditions of regularity of the method of summation are

$$
\sum_{k=0}^{n} \boldsymbol{r}_{k}=\sum_{k=0}^{n}\left|p_{k}\right|<c\left|P_{n}\right|
$$

and

$$
\left\{p_{n} / P_{n}\right\} \rightarrow 0 \text { as } n \rightarrow \infty
$$

3. If we write

$$
S_{n}(x)=\frac{1}{\pi} \int_{0}^{\pi} \phi(t) \frac{\operatorname{Sin}(n+1 / 2) t}{t} d t
$$

then we have 


$$
\begin{aligned}
\sigma_{n}(x) & =\frac{1}{\pi P(n)} \int_{0}^{\pi} \phi(t)\left(\sum_{k=0}^{n} p_{n-k} \frac{\operatorname{Sin}(k+1 / 2) t}{t}\right) d t \\
& =\int_{0}^{\pi} \phi(t) N_{n}(t) d t \\
& =\left(\int_{0}^{\pi / n}+\int_{\pi / n}^{\delta}+\int_{\delta}^{\pi}\right) \phi(t) N_{n}(t) d t \\
& =I_{1}+I_{2}+I_{3}, \quad \text { say }
\end{aligned}
$$

where $\delta$ is fixed.

Hence

$$
\begin{aligned}
I_{2} & =\frac{1}{\pi P(n)} \int_{\pi / n}^{\delta} \frac{\phi(t)}{t} \sum_{k=0}^{n}\left(p_{k} \operatorname{Sin}\left(n-k+\frac{1}{2}\right) t\right) d t \\
& =\frac{1}{\pi P(n)} \int_{\pi / n}^{\delta} \frac{\phi(t)}{t} \sum_{k=0}^{n} p_{k}\left\{\operatorname{Sin}\left(n+\frac{1}{2}\right) t \cdot \cos k t-\cos \left(n+\frac{1}{2}\right) t \cdot \operatorname{Sin} k t\right\} d t \\
\text { (3.3) } & =I_{2,1}-I_{2,2} \quad \text { say. }
\end{aligned}
$$

Now, if we write

$$
\begin{aligned}
I_{2,1}= & \frac{1}{\pi P(n)} \int_{\pi / n}^{\delta} \frac{\phi(t)}{t} \operatorname{Sin}\left(n+\frac{1}{2}\right) t \cdot\left\{\sum_{k t \leq 1}+\sum_{k t>1}\right\} p_{k} \cos k t \cdot d t \\
= & \frac{1}{\pi P(n)} \int_{\pi / n}^{\delta} \frac{\phi(t)}{t} \operatorname{Sin}\left(n+\frac{1}{2}\right) t \sum_{k t \leq 1} p_{k} \cos k t d t \\
& +\frac{1}{\pi P(n)} \int_{\pi / n}^{\delta} \frac{\phi(t)}{t} \cdot \operatorname{Sin}\left(n+\frac{1}{2}\right) t \sum_{k t>1} p_{k} \cos k t \cdot d t \\
= & \frac{1}{\pi P(n)} \int_{\pi / n}^{\delta} \frac{\phi(t)}{t} \operatorname{Sin}\left(n+\frac{1}{2}\right) t \sum_{1} d t \\
& +\frac{1}{\pi P(n)} \int_{\pi / n}^{\delta} \frac{\phi(t)}{t} \operatorname{Sin}\left(n+\frac{1}{2}\right) t \Sigma_{2} d t \\
(3.5) \quad & I_{2,1,1}+I_{2,1,2}, \quad \text { say. }
\end{aligned}
$$

4. We shall require the following lemma.

LEMMA. If we write

$$
\left|p_{n}\right|=r_{n}, \quad R_{n}=r_{0}+r_{1}+r_{2}+\cdots+r_{n}
$$

and

$$
r(u)=r_{[u]}, \quad R(u)=R_{[u]}
$$

where $[u]$ denotes the integer (largest) $\leqq u$, and

$$
V_{0} \equiv 0, \quad V_{n} \equiv \sum_{k=0}^{n}\left|p_{k}-p_{k-1}\right|, \quad V_{u} \equiv V_{[u]}
$$


then we have, from (3.4),

$$
\Sigma_{1}>P_{t} \cos 1>\frac{1}{2} P\left(\frac{1}{t}\right)
$$

and

$$
\left|\Sigma_{2}\right|=\frac{A}{t}\left\{r\left(\frac{1}{t}\right)+r(n)+V(n)-V\left(\frac{1}{t}-1\right)\right\} .
$$

This is known Hille and Tamarkin [3].

5. Now we shall prove the theorem.

Proof. Since

$$
\begin{aligned}
I_{1} & =\frac{1}{\pi} \int_{0}^{\pi / n} \phi(t) N_{n}(t) d t \\
& =\frac{1}{\pi} \int_{0}^{\pi / n}|\phi(t)| O(n) d t \\
& =O(n)\left[\bar{\Phi}_{1}(t)\right]_{0}^{\pi / n} \quad \text { by }(1.9) \\
& =O(n)\left[0\left\{t /\left(\log \frac{1}{t}\right)^{r}\right\}\right]_{0}^{\pi / n} \\
& =0(1), \quad \text { as } n \rightarrow \infty .
\end{aligned}
$$

From (3.5) and Lemma, above, we have

$$
\begin{aligned}
I_{2,1,1} & =\frac{1}{\pi P(n)} \int_{\pi / n}^{\delta} \frac{\phi(t)}{t} \operatorname{Sin}\left(n+\frac{1}{2}\right) t P\left(\frac{1}{t}\right) d t \\
& =\frac{1}{\pi P(n)} \int_{\pi / n}^{\delta} \frac{\phi(t)}{t} \operatorname{Sin} n t P\left(\frac{1}{t}\right) d t+o(1),
\end{aligned}
$$

by the regularity of the method of summation.

$$
\begin{aligned}
= & -\frac{1}{\pi P(n)} \int_{0}^{\delta-\pi / n} \frac{\phi(t+\pi / n)}{(t+\pi / n)} P\left(\frac{1}{t+\pi / n}\right) \operatorname{Sin} n t d t+o(1) \\
= & \frac{1}{2 \pi P(n)} \int_{\pi / n}^{\delta} \frac{\phi(t)}{t} \operatorname{Sin} n t P\left(\frac{1}{t}\right) d t-\frac{1}{2 \pi P(n)} \int_{0}^{\delta-\pi / n} \frac{\phi(t+\pi / n)}{t+\pi / n} \operatorname{Sin} n t \\
& \cdot P\left(\frac{1}{t+\pi / n}\right) d t+o(1) \\
= & \frac{1}{2 \pi P(n)} \int_{\pi / n}^{\delta-\pi / n} \frac{\phi(t)}{t} \operatorname{Sin} n t P\left(\frac{1}{t}\right) d t+\frac{1}{2 \pi P(n)} \int_{\delta-\pi / n}^{\delta} \frac{\phi(t)}{t} \operatorname{Sin} n t \\
& \cdot P\left(\frac{1}{t}\right) d t-\frac{1}{2 \pi P(n)} \int_{0}^{\pi / n} \frac{\phi(t+\pi / n)}{(t+\pi / n)} \operatorname{Sin} n t P\left(\frac{1}{t+\pi / n}\right) d t \\
& -\frac{1}{2 \pi P(n)} \int_{\pi / n}^{\delta-\pi / n} \frac{\phi(t+\pi / n)}{(t+\pi / n)} \operatorname{Sin} n t P\left(\frac{1}{t+\pi / n}\right) d t+o(1)
\end{aligned}
$$




$$
\begin{aligned}
& =\frac{1}{2 \pi P(n)} \int_{\pi / n}^{\delta-\pi / n}\left\{\frac{\phi(t)}{t} P\left(\frac{1}{t}\right)-\frac{\phi(t+\pi / n)}{t+\pi / n} P\left(\frac{1}{t+\pi / n}\right)\right\} \operatorname{Sin} n t d t \\
& -\frac{1}{2 \pi P(n)} \int_{0}^{\pi / n} \frac{\phi(t+\pi / n)}{t+\pi / n} \operatorname{Sin} n t P\left(\frac{1}{t+\pi / n}\right) d t \\
& +\frac{1}{2 \pi P(n)} \int_{\delta-\pi / n}^{\delta} \frac{\phi(t)}{t} P\left(\frac{1}{t}\right) \operatorname{Sin} n t d t+o(1) \\
& =\frac{1}{2 \pi P(n)} \int_{\pi / n}^{\delta-\pi / n}\left[\left\{\frac{\phi(t)}{t} P\left(\frac{1}{t}\right)-\frac{\phi(t+\pi / n)}{t} P\left(\frac{1}{t}\right)\right\}\right. \\
& +\left\{\frac{\phi(t+\pi / n)}{t} P\left(\frac{1}{t}\right)-\frac{\phi(t+\pi / n)}{t} P\left(\frac{1}{t+\pi / n}\right)\right\} \\
& \left.+\left\{\frac{\phi(t+\pi / n)}{t} P\left(\frac{1}{t+\pi / n}\right)-\frac{\phi(t+\pi / n)}{t+\pi / n} P\left(\frac{1}{t+\pi / n}\right)\right\}\right] \operatorname{Sin} n t d t \\
& -\frac{1}{2 \pi P(n)} \int_{0}^{\pi / n} \frac{\phi(t+\pi / n)}{t+\pi / n} \operatorname{Sin} n t P\left(\frac{1}{t+\pi / n}\right) d t \\
& +\frac{1}{2 \pi P(n)} \int_{\delta-\pi / n}^{\delta} \frac{\phi(t)}{t} \operatorname{Sin} n t P\left(\frac{1}{t}\right) d t+o(1) \\
& =\frac{1}{2 \pi P(n)}\left(\int _ { \pi / n } ^ { \delta - \pi / n } \left\{\frac{[\phi(t)-\phi(t+\pi / n)]}{t} P\left(\frac{1}{t}\right) d t\right.\right. \\
& +\left[P\left(\frac{1}{t}\right)-P\left(\frac{1}{t+\pi / n}\right)\right] \frac{\phi(t+\pi / n)}{t} \\
& \left.+P\left(\frac{1}{t+\pi / n}\right) \phi(t+\pi / n) \frac{\pi / n}{t(t+\pi / n)}\right\} \operatorname{Sin} n t d t \\
& -\int_{0}^{\pi / n} \frac{\phi(t+\pi / n)}{t+\pi / n} \operatorname{Sin} n t P\left(\frac{1}{t+\pi / n}\right) d t \\
& \left.+\int_{\delta-\pi / n}^{\delta} \frac{\phi(t)}{t} \operatorname{Sin} n t P\left(\frac{1}{t}\right) d t\right) \\
& +o(1) \\
& \text { (5.2) }=\left(P_{1}+P_{2}+P_{3}\right)+P_{4}+P_{5}, \text { say. }
\end{aligned}
$$

By virtue of (1.10) we have

$$
P_{1}=o(1), \text { as } n \rightarrow \infty .
$$

Also

$$
P_{2}=\frac{1}{2 \pi P(n)} \int_{\pi / n}^{\delta-\pi / n} \frac{\phi(t+\pi / n)}{t}\left\{P\left(\frac{1}{t}\right)-P\left(\frac{1}{t+\pi / n}\right)\right\} \operatorname{Sin} n t d t .
$$

Since, for all $0<1 / \alpha<1 / \beta$, we have

$$
P\left(\frac{1}{\beta}\right)-P\left(\frac{1}{\alpha}\right)=\int_{1 / \alpha}^{1 / \beta} p(\beta) d s+O\left\{p\left(\frac{1}{\alpha}\right)+p\left(\frac{1}{\beta}\right)\right\} .
$$




\section{Hence}

$$
\begin{aligned}
P_{2}= & O\left(\frac{1}{P(n)}\right)\left\{\int_{\pi / n}^{\delta}|\phi(t+\pi / n)| \frac{d t}{t} \int_{1 /(t+\pi / n)}^{1 / t} r(s) d s\right\} \\
& +O\left(\frac{1}{P(n)} \int_{\pi / n}^{\delta}|\phi(t+\pi / n)| r\left(\frac{1}{t}\right) \frac{d t}{t}\right) \\
& +O\left(\frac{1}{P(n)} \int_{\pi / n}^{\delta}|\phi(t+\pi / n)| r\left(\frac{1}{t+\pi / n}\right) \frac{d t}{t}\right) \\
= & P_{2,1}+P_{2,2}+P_{2,3} \text { say. }
\end{aligned}
$$

Now

$$
\begin{aligned}
P_{2,1}= & O\left(\frac{1}{P(n)}\right)\left\{\bar{\Phi}_{1}(t+\pi / n) \frac{1}{t} \int_{1 /(t+\pi / n)}^{1 / t} r(s) d s\right\}_{x / n}^{\delta} \\
& +O\left(\frac{1}{P(n)}\right) \int_{\pi / n}^{\delta} \bar{\Phi}_{1}(t+\pi / n) \frac{d t}{t^{2}} \int_{1 /(t+\pi / n)}^{1 / t} r(s) d s \\
& +O\left(\frac{1}{P(n)}\right) \int_{\pi / n}^{\delta} \bar{\Phi}_{1}(t+\pi / n) \frac{1}{t} r\left(\frac{1}{t}\right) \frac{d t}{t^{2}} \\
= & P_{2,1,1}+P_{2,1,2}+P_{2,1,3}, \text { say. }
\end{aligned}
$$

\section{We have}

$$
\begin{aligned}
P_{2,1,1}= & O\left(\frac{1}{P(n)}\right)\left\{o\left(\frac{1}{(\log 1 / t)^{r}}\right) \int_{1 /(t+\pi / n)}^{1 / t} r(s) d s\right\}_{x / n}^{\delta} \text { by (1.9) } \\
= & o\left(\frac{1}{P(n)}\right)\left\{\frac{1}{(\log n)^{r}} \int_{n / 2 x}^{n / \pi} r(s) d s\right\} \\
& +o\left(\frac{1}{P(n)}\right)\left\{\frac{1}{(\log 1 / \delta)^{r}} \int_{1 /(\delta+\pi / n)}^{1 / \delta} r(s) d s\right\} \\
= & o(1), \text { as } n \rightarrow \infty, \text { by (1.8). }
\end{aligned}
$$

And

$$
\begin{aligned}
P_{2,1,2} & =O\left(\frac{1}{P(n)}\right) \int_{x / n}^{\delta} o\left(\frac{t}{(\log 1 / t)^{r}}\right) \frac{d t}{t^{2}} \int_{1 /(t+\pi / n)}^{1 / t} r(s) d s \\
& =o\left(\frac{1}{P(n)}\right) \int_{\pi / n}^{\delta} \frac{d t}{t(\log 1 / t)^{r}} \int_{1 /(t+\pi / n)}^{1 / t} r(s) d s \\
& =o\left(\frac{1}{P(n)}\right) \int_{1 /(\delta+\pi / n)}^{n / \pi} r(s) d s \int_{(1 / s)-\pi / n}^{1 / s} \frac{d t}{t(\log 1 / t)^{r}}+o(1),
\end{aligned}
$$

by change of order of integration.

$$
\begin{aligned}
& =o\left(\frac{1}{P(n)}\right) \int_{1 /(\delta+\pi / n)}^{n / \pi} \frac{r(s) d s}{(\log 1 / s)^{r}} \int_{(1 / s)-\pi / n)}^{1 / s} \frac{d t}{t}+o(1) \\
& =o\left(\frac{1}{P(n)}\right) \int_{1 /(\delta+\pi / n)}^{n / \pi} \frac{r(s) d s}{(\log 1 / s)^{r}}(s \pi / n)+o(1)
\end{aligned}
$$




$$
\begin{aligned}
& =o\left(\frac{1}{P(n)}\right) \sum_{k=0}^{n} \frac{r(k)}{(\log (k+1))^{r}}+o(1) \\
& =o(1), \text { as } n \rightarrow \infty, \text { by }(1.8) .
\end{aligned}
$$

Finally, considering $P_{2,1,3}$, we have

$$
\begin{aligned}
P_{2,1,3} & =O\left(\frac{1}{P(n)}\right) \int_{x / n}^{\delta} o\left(\frac{t}{(\log 1 / t)^{r}}\right) \frac{d t}{t} r\left(\frac{1}{t}\right) \frac{1}{t^{2}}, \quad \text { by (1.9) } \\
& =o\left(\frac{1}{P(n)}\right) \int_{x / n}^{\delta} \frac{r(1 / t)}{(\log 1 / t)^{r}} \frac{d t}{t^{2}} \\
& =o\left(\frac{1}{P(n)}\right) \int_{n / x}^{1 / \delta} \frac{r(s)}{(\log s)^{r}} d s \\
& =o\left(\frac{1}{P(n)}\right) \sum_{k=0}^{n} r(k) /\{\log (k+1)\}^{r} \\
& =o(1) \quad \text { as } n \rightarrow \infty, \text { by (1.8). }
\end{aligned}
$$

Thus from (5.5), (5.6), (5.7) and (5.8) we see that

$$
P_{2,1}=o(1) \text { as } n \rightarrow \infty \text {. }
$$

Estimating $P_{2,2}$ we find that

$$
\begin{aligned}
P_{2,2}= & O\left(\frac{1}{P(n)}\right) \int_{x / n}^{\delta}|\phi(t+\pi / n)| r\left(\frac{1}{t}\right) d t \\
= & O\left(\frac{1}{P(n)}\right)\left\{\left[\bar{\Phi}_{1}(t) r\left(\frac{1}{t}\right) \frac{1}{t}\right]_{x / n}^{\delta}+\int_{x / n}^{\delta} \bar{\Phi}_{1}(t) r\left(\frac{1}{t}\right) \frac{d t}{t^{2}}\right. \\
& \left.-\int_{x / n}^{\delta} \bar{\Phi}_{1}(t) \frac{1}{t} d r\left(\frac{1}{t}\right)\right\}+o(1) .
\end{aligned}
$$

Here, the integrated part is $o(1)$, by virtue of (1.7) and the fact that $P(n) \rightarrow \infty$ as $n \rightarrow \infty$. The second part is

$$
\begin{aligned}
& o\left(\frac{1}{P(n)}\right) \int_{x / n}^{\delta} o\left(\frac{1}{(\log 1 / t)^{r}}\right) r\left(\frac{1}{t}\right) \frac{d t}{t}, \quad \text { by }( \\
& \quad=o\left(\frac{1}{P(n)}\right) \int_{n / x}^{1 / \delta} \frac{r(s)}{s(\log s)^{r}} d s \\
& \quad=o(1), \quad \text { by }(1.8)
\end{aligned}
$$

The third term is

$$
\begin{aligned}
& O\left(\frac{1}{P(n)}\right) \int_{x / n}^{\delta} o\left(\frac{t}{(\log 1 / t)^{r}}\right) \frac{1}{t} d r\left(\frac{1}{t}\right) \\
& \quad=o\left(\frac{1}{P(n)}\right) \sum_{k=0}^{n} \frac{\left|r_{k}-r_{k-1}\right|}{(\log (k+1))^{r}} \\
& \quad=o(1) \text { as } n \rightarrow \infty, \text { by }(1.8) .
\end{aligned}
$$


Thus we see that

$$
P_{2,2}=o(1) \quad \text { as } \quad n \rightarrow \infty .
$$

Similarly, we can show that

$$
P_{2,3}=o(1) \quad \text { as } n \rightarrow \infty .
$$

Hence by (5.4), (5.9), (5.10) and (5.11) we get

$$
P_{2}=o(1) \text { as } n \rightarrow \infty \text {. }
$$

Evaluating $P_{3}$ we have

$$
\begin{aligned}
P_{3} & =O\left(\frac{\pi}{n P(n)}\right) \int_{\pi / n}^{\delta}|\phi(t+\pi / n)| P\left(\frac{1}{t+\pi / n}\right) \frac{d t}{t(t+\pi / n)} \\
& =O\left(\frac{1}{n}\right) \int_{\pi / n}^{\delta}|\phi(t+\pi / n)| \frac{d t}{t^{2}} \\
& =O\left(\frac{1}{n}\right)\left[\left\{\bar{\Phi}_{1}(t+\pi / n) \frac{1}{t^{2}}\right\}_{\pi / n}^{\delta}+2 \int_{\pi / n}^{\delta} \bar{\Phi}_{1}(t+\pi / n) \frac{d t}{t^{3}}\right] \\
& =o\left(\frac{1}{n}\right)+o\left(\frac{1}{(\log n)^{r}}\right) \text { by (1.9) } \\
(5.13) \quad & o(1), \text { as } n \rightarrow \infty .
\end{aligned}
$$

And

$$
\begin{aligned}
P_{4} & =\frac{1}{2 \pi P(n)} \int_{0}^{\pi / n} \phi(t+\pi / n) \operatorname{Sin} n t P\left(\frac{1}{t+\pi / n}\right) \frac{d t}{t+\pi / n} \\
& =-\frac{1}{2 \pi P(n)} \int_{\pi / n}^{2 \pi / n} \phi(t) \operatorname{Sin} n t P\left(\frac{1}{t}\right) \frac{d t}{t} \\
& =O\left(\frac{1}{P(n)}\right) \int_{\pi / n}^{2 \pi / n}|\phi(t)| O(n t) P\left(\frac{1}{t}\right) \frac{d t}{t} \\
& =O(n) \int_{\pi / n}^{2 \pi / n}|\phi(t)| d t \\
& =o\left(\frac{1}{(\log n)^{r}}\right), \quad \text { by (1.9) } \\
& =o(1) \text { as } n \rightarrow \infty .
\end{aligned}
$$

Also

$$
\begin{aligned}
P_{5} & =O\left(\frac{1}{P(n)}\right) \int_{\delta-\pi / n}^{\delta}|\phi(t)| P\left(\frac{1}{t}\right) \frac{d t}{t} \\
& =o(1),
\end{aligned}
$$

by the regularity of the method of summation and since the interval $(\delta-\pi / n, \delta)$ tends to zero as $n \rightarrow \infty$. 
Consequently from (5.2), (5.3), (5.12), (5.13), (5.14) and (5.15) we have

$$
I_{2,1,1}=o(1), \text { as } n \rightarrow \infty
$$

Now

$$
\begin{aligned}
I_{2,1,2}= & \frac{1}{\pi P(n)} \int_{x / n}^{\delta} \frac{\phi(t)}{t} \operatorname{Sin}\left(n+\frac{1}{2}\right) t \Sigma_{2} d t \\
= & O\left(\frac{1}{P(n)}\right) \int_{x / n}^{\delta} \frac{|\phi(t)|}{t}\left\{\frac{r(1 / t)}{t}+\frac{r(n)}{t}\right. \\
& \left.+\frac{1}{t}\left[V(n)-V\left(\frac{1}{t}-1\right)\right]\right\} d t, \quad \text { by (4.5) } \\
= & Q_{1}+Q_{2}+Q_{3}, \text { say. }
\end{aligned}
$$

We have

$$
\begin{aligned}
Q_{1}= & O\left(\frac{1}{P(n)}\right) \int_{\pi / n}^{\delta} \frac{|\phi(t)|}{t^{2}} r\left(\frac{1}{t}\right) d t \\
= & O\left(\frac{1}{P(n)}\right)\left\{\left[o\left(\frac{t}{\{\log 1 / t\}^{r}}\right) r\left(\frac{1}{t}\right) \frac{1}{t^{2}}\right]_{x / n}^{\delta}+\int_{x / n}^{\delta} o\left(\frac{t}{\{\log 1 / t\}^{r}}\right) r\left(\frac{1}{t}\right) \frac{d t}{t^{3}}\right. \\
& \left.+\int_{\pi / n}^{\delta} o\left(\frac{t}{\{\log 1 / t\}^{r}}\right) \frac{1}{t^{2}} d r\left(\frac{1}{t}\right)\right\} \text { by (1.9). }
\end{aligned}
$$

Here the integrated part is $o(1)$, by $(1.7)$ and the fact that $P(n) \rightarrow$ $\infty$ as $n \rightarrow \infty$. Also the second term is

$$
\begin{aligned}
o\left(\frac{1}{P(n)}\right) \int_{x / n}^{\delta} r\left(\frac{1}{t}\right) \frac{d t}{t^{2}} \frac{1}{(\log 1 / t)^{r}} \\
\quad=o\left(\frac{1}{P(n)}\right) \int_{n / x}^{1 / \delta} \frac{r(s)}{(\log s)^{r}} d s \\
\quad=o\left(\frac{1}{P(n)}\right) \sum_{k=0}^{n} \frac{k\left|p_{k}-p_{k-1}\right|}{(\log (k+1))^{r}} \\
=o(1) \quad \text { by }(1.8) .
\end{aligned}
$$

The third part is

$$
\begin{aligned}
& o\left(\frac{1}{P(n)}\right) \int_{n / x}^{1 / \delta} \frac{s d r(s)}{(\log s)^{r}} \\
& \quad=o\left(\frac{1}{P(n)}\right) \sum_{k=0}^{n} \frac{k\left|p_{k}-p_{k-1}\right|}{(\log (k+1))^{r}} \\
& \quad=o(1) \text { as } n \rightarrow \infty, \text { by (1.8). }
\end{aligned}
$$

Thus we see that

$$
Q_{1}=o(1) \text { as } n \rightarrow \infty .
$$


Now

$$
\begin{aligned}
Q_{2}= & O\left(\frac{r(n)}{P(n)}\right) \int_{\pi / n}^{\delta} \frac{|\phi(t)|}{t^{2}} d t \\
= & O\left(\frac{r(n)}{P(n)}\right)\left\{\left(\frac{\bar{\Phi}_{1}(t)}{t^{2}}\right)_{\pi / n}^{\delta}+2 \int_{\pi / n}^{\delta} \bar{\Phi}_{1}(t) \frac{d t}{t^{3}}\right\} \\
= & o\left(\frac{r(n)}{P(n)}\right)+o\left(\frac{n r(n)}{P(n)(\log n)^{r}}\right) \\
& +o\left(\frac{r(n)}{P(n)}\right) \int_{\pi / n}^{\delta} \frac{d t}{t^{2}(\log 1 / t)^{r}} \\
= & o\left\{\frac{(\log n)^{r}}{n}\right\}+o(1)+o\left\{\frac{r(n) n}{P(n)(\log n)^{r}}\right\} \\
= & o(1)+o\left(\frac{1}{n}\right) \\
= & o(1) \quad \text { as } \quad n \rightarrow \infty .
\end{aligned}
$$

Lastly

$$
\begin{aligned}
Q_{3}= & O\left(\frac{1}{P(n)} \int_{\pi / n}^{\delta} \frac{|\phi(t)|}{t^{2}}\left[V(n)-V\left(\frac{1}{t}-1\right)\right] d t\right. \\
= & O\left(\frac{1}{P(n)}\right)\left\{\left[\frac{\bar{\Phi}_{1}(t)}{t^{2}}\left(V(n)-V\left(\frac{1}{t}-1\right)\right)\right]_{x / n}^{\delta}\right. \\
& +2 \int_{\pi / n}^{\delta} \frac{\bar{\Phi}_{1}(t)}{t^{3}}\left[V(n)-V\left(\frac{1}{t}-1\right)\right] d t \\
& \left.-\int_{\pi / n}^{\delta} \frac{\bar{\Phi}_{1}(t)}{t^{2}} d V\left(\frac{1}{t}-1\right)\right\} .
\end{aligned}
$$

The integrated part is $o(1)$, by (1.7) and the fact that

$$
W_{n} \equiv \sum_{k=0}^{n} \frac{k\left|p_{k}-p_{k-1}\right|}{(\log (k+1))^{r}} ; \quad W_{0} \equiv 0 .
$$

Then, by (1.8) we have

$$
\begin{aligned}
V_{n} & =\sum_{k=0}^{n}\left|p_{k}-p_{k-1}\right| \\
& =\sum_{k=0}^{n} \frac{\{\log (k+1)\}^{r}}{k}\left(W_{k}-W_{k-1}\right) \\
& =\sum_{k=0}^{n-1} W_{k}\left\{4\left[\frac{\left\{\log (k+1\}^{r}\right.}{k}\right]\right\}+\frac{W_{n}\{\log (n+1)\}}{n} \\
& =o\{R(n)\} .
\end{aligned}
$$

Now the second term is 


$$
\begin{aligned}
o\left[\frac{1}{P(n)}\right. & \left.\int_{x / n}^{\delta}\left\{V(n)-V\left(\frac{1}{t}-1\right)\right\} \frac{d t}{t^{2}(\log 1 / t)^{r}}\right] \\
= & o\left[\frac{1}{P(n)} \int_{1 / \delta}^{n / x} \frac{d s}{(\log s)^{r}}[V(n)-V(s-1)]\right] \\
= & o\left\{\frac{1}{P(n)} \int_{0}^{n} \frac{s}{\{\log (s+1)\}^{r}} d V(s)\right\} \\
& +o\left[\frac{1}{R(n)}\{V(n)-V(s-1)\} \frac{s}{\{\log (s+1)\}^{r}}\right]_{0}^{n} \\
= & o\left\{\frac{1}{P(n)} \sum_{k=0}^{n} \frac{k\left|p_{k}-p_{k-1}\right|}{\{\log (k+1)\}^{r}}\right\}+o\left\{\frac{1}{P(n)} \frac{n\left|p_{n}-p_{n-1}\right|}{\{\log (n+1)\}^{r}}\right\}
\end{aligned}
$$

which is $o(1)$, by virtue of (1.7), (1.8) and the fact that $V_{n}=o\{P(n)\}$. The third term is

$$
\begin{gathered}
o\left\{\frac{1}{P(n)} \int_{x / n}^{\delta} \frac{1}{t(\log 1 / t)^{r}}\left|d V\left(\frac{1}{t}-1\right)\right|\right\}, \quad \text { by (1.9) } \\
=o\left\{\frac{1}{P(n)}\right\} \int_{0}^{n} \frac{s|d V(s-1)|}{\{\log (s+1)\}^{r}}
\end{gathered}
$$

which is $o(1)$, as in the case of second term.

Thus we have

$$
Q_{3}=o(1) \text { as } n \rightarrow \infty .
$$

From (5.17), (5.18), (5.19) and (5.20), we have

$$
I_{2,1,2}=o(1) \text { as } n \rightarrow \infty .
$$

From (3.4) (3.5), (3.16) and (5.21), we see that

$$
I_{2,1}=o(1) \quad \text { as } n \rightarrow \infty \text {. }
$$

Similarly, we can show that

$$
I_{2,2}=o(1) \text { as } n \rightarrow \infty \text {. }
$$

From (3.3), (5.22) and (5.23) we get

$$
I_{2}=o(1) \text { as } n \rightarrow \infty \text {. }
$$

Lastly by Riemann Lebesgue Theorem and the regularity of the method of summation, we have, as $n \rightarrow \infty$

$$
I_{3}=o(1) \text {. }
$$

Collection of (3.2), (5.24) and (5.25) as $n \rightarrow \infty$, completes the proof of the theorem.

I am much indebted to Professor M. L. Misra for his valuable guidance during the preparation of this paper. 


\section{REFERENCES}

1. M. Astrachan, Studies in the summability of Fourier Series by Nörlund means, Duke Math. J. 2 (1936), 543-568.

2. E. Hille and J. D. Tamarkin, On the summability of Fourier Series. Proc. Nat. Acad. Sci., 14 (1928), 915-918.

3. - On the summability of Fourier Series. Trans. Amer. Math. Soc., 34 (1932), 757-783.

4. N. E. Nörlund, Sur une application des fonctions permutables, Londs. Universitets Arsskrift, (N.F.) avd. 2, 16 No. 3 (1920).

5. O. P. Varshney, On the Nörlund summability of a Fourier Series and its conjugate series, Thesis presented for the degree of $\mathrm{Ph}$. D. at the University of Saugar (March, 1960).

6. G. F. Woronoi, Extension of the notion of the limit of the sum of terms of an infinite series, Proc. of 11th Congress of Russian Naturalists and Scientists (in Russian). St. Petersburg, 1902, 60-61. There is an annotated English translation by J. D. TamarkinAnn. Math., 33 (1932), 422-428.

College of EngG. ANd Tech.

NEW DELHI, INDIA 


\section{PACIFIC JOURNAL OF MATHEMATICS}

\section{EDITORS}

\section{RalPh S. Phillips}

Stanford University

Stanford, California

M. G. Arsove

University of Washington

Seattle 5, Washington
J. Dugundji

University of Southern California Los Angeles 7, California

Lowell J. Paige

University of California

Los Angeles 24, California

\section{ASSOCIATE EDITORS}
E. F. BECKENBACH
D. DERRY
H. L. ROYDEN
E. G. STRAUS
T. M. CHERRY
M. OHTSUKA
E. SPANIER
F. WOLF

\section{SUPPORTING INSTITUTIONS}

\author{
UNIVERSITY OF BRITISH COLUMBIA \\ CALIFORNIA INSTITUTE OF TECHNOLOGY \\ UNIVERSITY OF CALIFORNIA \\ MONTANA STATE UNIVERSITY \\ UNIVERSITY OF NEVADA \\ NEW MEXICO STATE UNIVERSITY \\ OREGON STATE UNIVERSITY \\ UNIVERSITY OF OREGON \\ OSAKA UNIVERSITY \\ UNIVERSITY OF SOUTHERN CALIFORNIA
}

STANFORD UNIVERSITY

UNIVERSITY OF TOKYO

UNIVERSITY OF UTAH

WASHINGTON STATE UNIVERSITY

UNIVERSITY OF WASHINGTON

AMERICAN MATHEMATICAL SOCIETY

CALIFORNIA RESEARCH CORPORATION SPACE TECHNOLOGY LABORATORIES

NAVAL ORDNANCE TEST STATION

Printed in Japan by International Academic Printing Co., Ltd., Tokyo Japan 


\section{Pacific Journal of Mathematics}

\section{Vol. 13, No. 1 \\ March, 1963}

Frantz Woodrow Ashley, Jr., A cone of super-(L) functions............. 1

Earl Robert Berkson, Some metrics on the subspaces of a Banach space....

Felix Earl Browder and Walter Strauss, Scattering for non-linear wave

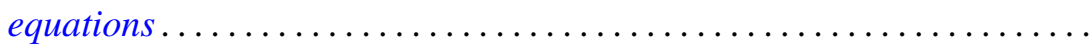

Edmond Darrell Cashwell and C. J. Everett, Formal power series ..........

Frank Sydney Cater, Continuous linear functionals on certain topological

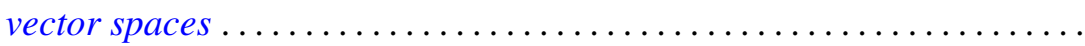

John Douglas Dixon, General group extensions ....................

Robert Pertsch Gilbert, On harmonic functions of four variables with

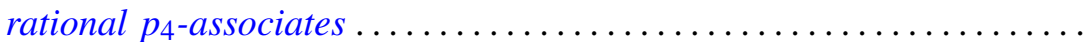

Irving Leonard Glicksberg, On convex hulls of translates ..............

Simon Hellerstein, On a class of meromorphic functions with deficient zeros

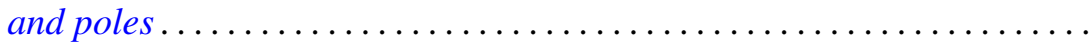

Donald William Kahn, Secondary cohomology operations which extend the

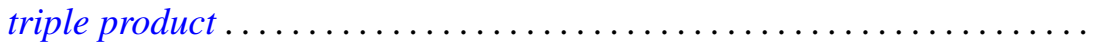

G. K. Leaf, A spectral theory for a class of linear operators .............

R. Sherman Lehman, Algebraic properties of the composition of solutions of partial differential equations ........................... 157

Joseph Lehner, On the generation of discontinuous groups ............. 169

S. P. Lloyd, On certain projections in spaces of continuous functions ...... 171 Fumi-Yuki Maeda, Generalized spectral operators on locally convex spaces ..................................

Donald Vern Meyer, $E^{3}$ modulo a 3-cell

William H. Mills, An application of linear programming to permutation groups.

Richard Scott Pierce, Centers of purity in abelian groups

Christian Pommerenke, On meromorphic starlike functions ...

Zalman Rubinstein, Analytic methods in the study of zeros of

polynomials...

B. N. Sahney, On the Nörlund summability of Fourier series

Tôru Saitô, Regular elements in an ordered semigroup . .

Lee Meyers Sonneborn, Level sets on spheres...........

Charles Andrew Swanson, Asymptotic estimates for limit point

problems .

Lucien Waelbroeck, On the analytic spectrum of Arens . .

Alvin (Murray) White, Singularities of a harmonic function of three

variables given by its series development .............

Kōichi Yamamoto, Decomposition fields of difference sets ...

Chung-Tao Yang, On the action of $\mathrm{SO}(3)$ on a cohomology manifold... 